Note

\title{
Universal tail sequence-SSR applied to molecular characterization of tropical maize
}

\section{hybrids}

\author{
Carlos Alexandre Gomes Ribeiro ${ }^{1}$, Marcos de Oliveira Pinto², Talles Eduardo Ferreira Maciel ${ }^{3}$, Maria Marta Pastina², Everaldo \\ Gonçalves de Barros ${ }^{4}$, Claudia Teixeira Guimarães ${ }^{2 *}$
}

${ }^{1}$ Federal University of Viçosa - Dept. of Genetics and Breeding, Av. PH Rolfs, s/n - 36570-000 - Viçosa, MG Brazil.

Embrapa Maize and Sorghum, Rod. MG 424, km 65 35701-970 - Sete Lagoas, MG - Brazil.

3Federal University of Viçosa/Institute of Biotechnology Applied to Agriculture.

${ }^{4}$ Catholic University of Brasília - Dept. of Genomic Sciences and Biotecnhology, SGAN, 916 - 70790-160 - Brasília, DF - Brazil.

${ }^{*}$ Corresponding author <claudia.guimaraes@embrapa.br>

Edited by: Roberto Fritsche Neto

Received March 02, 2016

Accepted April 16, 2016

\begin{abstract}
The development of efficient and low-cost genotyping methods is essential to precise genetic characterization of cultivars. Here, we present a system based on fluorescently labeled universal tail sequence primers (UTSP) to resolve microsatellite (SSR) markers as an alternative for molecular fingerprinting of maize. A set of 20 SSRs using the UTSP presented an average polymorphic information content of 0.84 , which provided a probability of random identity ranging from $10^{-7}$ to $10^{-14}$, and a minimum exclusion power of $99.99998 \%$ in a group of 48 tropical maize single-cross hybrids traded in Brazil. The genetic diversity analysis based on multidimensional scaling explained approximately $28 \%$ of the total variance for the first two coordinates, tending to group the hybrids according to their respective origin. Additionally, this genotyping system presented a high distinctiveness capacity, which is widely recommended for genetic purity and fingerprinting analyses. Thus, this marker system has a strong potential as a tool for complementary analysis of distinguishability, uniformity and stability required for cultivar registration.

Keywords: Zea mays, fingerprinting, genotyping, cultivar protection
\end{abstract}

\section{Introduction}

Maize is the most produced cereal in the world, and Brazil ranks as the third largest producer. Currently, over 2,500 maize cultivars, including inbred lines, single-cross hybrids, and varieties are registered in the National Cultivar Registration Service (MAPA, 2015). In 2014/2015, the Brazilian maize seed market accounted for over US\$ 1.3 billion, considering the retail seed price (APPS, 2015), thus emphasizing the strategic importance of improved maize cultivars to the national economy. The increasing number of registered maize cultivars hampers their distinction based on phenotypic analyses. Therefore, an accurate assessment of distinctiveness is needed for distinguishability, uniformity and stability (DUS) tests, which are officially required for cultivar registration in Brazil (MAPA, 2015). For this purpose, a set of morphological descriptors for each species has been defined (MAPA, 2015), which presents a number of limitations, such as late and subjective evaluation, environmental influence and reduced number of traits. Thus, molecular markers can be an alternative to complement the morphological evaluation, bringing a superior level of accuracy to DUS tests (Priolli et al., 2002).

Several studies have been proposed for including microsatellites or simple sequence repeat (SSR) markers as molecular descriptors for DUS tests in Brazil. These markers have been used to characterize banana (Jesus et al., 2009), essentially derived soybean cultivars (Rodrigues et al., 2008) and for genetic purity certification of maize hybrid seeds (Salgado et al., 2006). However, little informa- tion is available on a suitable combination of SSR markers to be applied as molecular descriptors for commercial maize hybrids in Brazil.

SSR is a relatively simple technique, but its low level of automation increases the costs and time of large-scale analyses. The inclusion of an extended fluorescently labeled tail in the SSR primers has been proposed to improve automation and reduce genotyping costs (Cryer et al., 2005; Arruda et al., 2010; Diniz et al., 2007; Hayden et al., 2008; Missiaggia and Grattapaglia, 2006; Ribeiro et al., 2013).

There is increasing demand for molecular fingerprinting of maize cultivars, especially as a result of the Plant Variety Protection law, which requires a rapid and cost-effective genotyping methodology. Thus, our current study aimed to validate a set of SSR markers using a universal tail sequence primers (UTSP) system in 48 Brazilian maize single-cross hybrids for future application in cultivar protection.

\section{Materials and Methods}

\section{Plant material}

We used 48 commercial maize single-cross hybrids that were available in the Brazilian seed market in 2011. The seeds of all cultivars were purchased in local markets. The maize cultivars were developed by eight private seed companies and by Embrapa Maize \& Sorghum, representing the public sector. Three single-cross hybrids from Embrapa were genotyped as well as their corresponding parental lines in order to confirm the origin of the alleles present in the hybrids. 


\section{DNA extraction and genotyping}

Genomic DNA was extracted using 96-well plates by the cetyl trimethylammonium bromide (CTAB) method (Saghai-Maroof et al., 1984). Leaf discs were collected from a bulk of 20 seedlings, representing each genotype. The DNA was quantified spectrophotometrically.

Polymerase chain reaction (PCR) was performed using UTSP as described by Ribeiro et al. (2013). This genotyping system was originally proposed by Oetting et al. (1995), which used a fluorescently labeled primer to anneal with a specific primer used to amplify the target fragments in PCR, labelling indirectly the amplified fragment. The UTSP system was composed of three primers: the sense microsatellite primer with 17 extra base pairs (bp) tail in the $5^{\prime}$ end, these additional sequences are identical to the universal primers T3 (ATTAACCCTCACTAAAG), T7 (AATACGACTCACTATAG) or M13 (GTAAAACGACGGCCAGT); the respective antisense microsatellite primer, and the universal primer with the same sequences of the sense primer tails, which can be T3, T7 or M13 labeled with each of the fluorochromes HEX, NED or 6-FAM, respectively. The amplification reaction $(20 \mu \mathrm{L})$ contained DNA (30 ng), 1X PCR buffer (10 mM Tris- $\mathrm{HCl}, \mathrm{pH} 8.8,50$ $\mathrm{mM} \mathrm{KCl}, 0.08 \%$ Nonidet P40), $1.5 \mathrm{mM} \mathrm{MgCl}_{2^{\prime}} 0.25 \mathrm{mM}$ dNTP, $0.025 \mu \mathrm{M}$ of sense primer, $0.125 \mu \mathrm{M}$ of antisense primer, $0.125 \mu \mathrm{M}$ of labeled universal primer and $1.0 \mathrm{U}$ of Taq DNA polymerase. Conventional amplification reactions were performed using the same conditions applied to the UTSP, except for the primers, which were at 0.125 $\mu \mathrm{M}$ for the regular sense and antisense SSR primers. The PCR for each microsatellite locus was performed separately and mixed before loading in the sequencer.
The amplification program consisted of an initial denaturation step of $94^{\circ} \mathrm{C}$ for $4 \mathrm{~min}$, followed by 8 cycles of denaturation at $94{ }^{\circ} \mathrm{C}$ for $40 \mathrm{~s}$, annealing at $68{ }^{\circ} \mathrm{C}$ for $40 \mathrm{~s}$ and extension at $72{ }^{\circ} \mathrm{C}$ for $40 \mathrm{~s}$, and 25 cycles of denaturation at $94{ }^{\circ} \mathrm{C}$ for $40 \mathrm{~s}$, annealing at $60{ }^{\circ} \mathrm{C}$ for $40 \mathrm{~s}$ and extension at $72{ }^{\circ} \mathrm{C}$ for $40 \mathrm{~s}$. The final extension step was set to $72{ }^{\circ} \mathrm{C}$ for $20 \mathrm{~min}$. A volume of $1.0 \mu \mathrm{L}$ of each PCR amplification product was mixed with $8.75 \mu \mathrm{L}$ of $\mathrm{Hi}-\mathrm{Di}$ formamide and $0.25 \mu \mathrm{L}$ of molecular size standard GeneScan 500 ROX (ThermoFisher Scientific Inc.). The samples were denatured for $3 \mathrm{~min}$ at $95{ }^{\circ} \mathrm{C}$ and maintained on ice until loading into the ABI $3100 \mathrm{XL}$ sequencer using POP4 polymer (ThermoFisher Scientific Inc.). The data were automatically collected and coded with the GeneMapper 3.5 software (ThermoFisher Scientific Inc.).

Out of 100 primers previously tested in a larger number of lines, we selected a set of 20 SSR loci well distributed along the maize genome and showing high polymorphic information content (PIC) values among the 48 maize single-cross hybrids. The sequences of all selected SSR primers and the respective universal primer associated with the fluorescence are shown in Table 1.

\section{Data analysis}

Each microsatellite allele was considered as a dominant marker, which was coded as 1 (presence) or 0 (absence). The PIC was calculated according to the expression:

$$
P I C=1-\sum_{j=1}^{n} P_{i j}^{2}
$$

where $P_{i j}^{2}$ is the frequency of the $j^{\text {th }}$ allele of the $i^{\text {th }}$ marker.

Table 1 - SSR markers, sense and antisense primer sequences, and their respective universal primer and fluorescence.

\begin{tabular}{|c|c|c|c|}
\hline Marker & Sense primer $\left(5^{\prime} \rightarrow 3^{\prime}\right)$ & Antisense primer $\left(5^{\prime} \rightarrow 3^{\prime}\right)$ & $\begin{array}{c}\text { Universal primer / } \\
\text { Fluorescence }\end{array}$ \\
\hline bnlg182 & GTAAAACGACGGCCAGTAGACCATATTCCAGGCTTTACAG & ACAACTAGCAGCAGCACAAGG & M13/6FAM \\
\hline bnlg2241 & GTAAAACGACGGCCAGTTGCACACTCTCTTGCATCG & TAGTCAGCATCTGCCGTGTC & M13/6FAM \\
\hline bnlg589 & GTAAAACGACGGCCAGTGGGTCGTTTAGGGAGGCACCTTTGGT & GCGACAGACAGACAGACAAGCGCATTGT & M13/6FAM \\
\hline phi116 & GTAAAACGACGGCCAGTGCATACGGCCATGGATGGGA & TCCCTGCCGGGACTCCTG & M13/6FAM \\
\hline umc1016 & GTAAAACGACGGCCAGTGTGATACCGGGTAATCTGGTGC & GATGATGGGTGATCATCGGTTC & M13/6FAM \\
\hline umc1033 & GTAAAACGACGGCCAGTCTTCTTCGTAAAGGCATTTTGTGC & GTGCGGGATTCCTTAGTTTGC & M13/6FAM \\
\hline umc1862 & GTAAAACGACGGCCAGTATGGGCACATGAAAAAGAGACATT & CCCATGAGAAGAGTGAAGACAACA & M13/6FAM \\
\hline bnlg1006 & ATTAACCCTCACTAAAGGACCAGCGTGTTGATCCC & GGAGACCCCGACTCTCTCTC & T3/HEX \\
\hline bnlg125 & ATTAACCCTCACTAAAGGGGACAAAAGAAGAAGCAGAG & GAAATGGGACAGAGACAGACAAT & T3/HEX \\
\hline bnlg161 & ATTAACCCTCACTAAAGGCTTTCGTCATACACACACATTCA & ATGGAGCATGAGCTTGCATATTT & T3/HEX \\
\hline bnlg1863 & ATTAACCCTCACTAAAGGGCGTTCGTTTTGCACTAAT & CGACACAGTTGACATCAGGG & T3/HEX \\
\hline umc1008 & ATTAACCCTCACTAAAGTCTAGCTTGTGGTGGTGGTTGA & ACATGAGCACAAAGACTGACGC & T3/HEX \\
\hline umc1653 & ATTAACCCTCACTAAAGGAGACATGGCAGACTCACTGACA & GCCGCCCACGTACATCTATC & T3/HEX \\
\hline bnlg1338 & AATACGACTCACTATAGGTGCAGAATGCAGGCAATAG & GCAAATGTTTTCACACACACG & T7/NED \\
\hline dupssr14 & AATACGACTCACTATAGAGCAGGTACCACAATGGAG & GTGTACATCAAGGTCCAGATTT & T7/NED \\
\hline dupssr24 & AATACGACTCACTATAGACTGCACTGCACCTCTCTC & ACACAACGGCTTCTAACCTT & T7/NED \\
\hline phi053 & AATACGACTCACTATAGCTGCCTCTCAGATTCAGAGATTGAC & AACCCAACGTACTCCGGCAG & T7/NED \\
\hline phi084 & AATACGACTCACTATAGAGAAGGAATCCGATCCATCCAAGC & CACCCGTACTTGAGGAAAACCC & T7/NED \\
\hline umc1019 & AATACGACTCACTATAGCCAGCCATGTCTTCTCGTTCTT & AAACAAAGCACCATCAATTCGG & T7/NED \\
\hline umc1084 & AATACGACTCACTATAGGATAAAAAGGCAAGTGCAACAAGG & ATATCAACCAGAGGCTGGAACTTG & T7/NED \\
\hline
\end{tabular}

${ }^{*}$ The sequence underlined in the sense primers was included in each respective fluorescently labeled universal primer. 
A genetic dissimilarity matrix was calculated as proposed by Nei and Li (1979) using the software Genes version 2009.7.0. A classical multidimensional scaling (MDS) analysis was performed based on the dissimilarity matrix using the cmdscale function available in the $\mathrm{R}$ package (R-project, 2015), and a biplot for the first two coordinates was generated.

The probability of random identity (PRI) for all SSR markers was calculated as follows:

$P R I=\prod_{i=1}^{n} P_{i}$

where $P_{i}$ is the frequency of allele $i$ and $n$ is the number of evaluated markers.

Based on the PRI values, the percentage of excluding power $(\mathrm{EP})$ was calculated as follows: $E P=(1-P R I)$ $\times 100$, which indicates the power to unambiguously identify a given hybrid among all hybrids in the set, using the 20 SSR markers described in this study.

\section{Results and Discussion}

\section{Standardization of the universal tail sequence primer method}

The alleles amplified using the UTSP method exhibited a difference of $17 \mathrm{bp}$, corresponding to the extended tail, in comparison with the amplified product using the conventional SSR primers. For example, in the parental line A, marker umc1016 amplified 114 and 97 bp-alleles by the UTSP and conventional SSR primers, respectively (Table 2). However, the alleles amplified with primer umc1653 presented a difference of $16 \mathrm{bp}$ instead of the expected $17 \mathrm{bp}$ (Table 2). Despite the high accuracy of the capillary electrophoresis system, small variations of up to two bp in the allele sizes can be expected. The alleles amplified in the parental lines were also confirmed in their respective single-cross hybrids, using both UTSP and conventional methods (Table 2). Additionally, the UTSP method showed less amplification artifacts than the conventional SSR primers. Similar results were also obtained using 13 SSR based on UTSP to evaluate the genetic diversity of 30 soybean cultivars (Ribeiro et al., 2013).

Although maize is a diploid $(2 n=2 x=20)$ species, up to four alleles per locus could be observed in a single- cross hybrid, due to the residual heterozygosity in both parental inbred lines. In fact, two alleles were amplified in parental line A for umc1653, and were confirmed by both genotyping methods (Table 2). According to Liu et al. (2003), approximately $4 \%$ of the 33,900 SSR genotypes generated more than one amplified band per maize inbred line, which could be due to residual heterozygosity, contamination, or the amplification of similar sequences in two different genomic regions. The minor allele frequency expected in a maize single-cross hybrid per locus would be 0.25 , considering that both parental inbred lines are heterozygous for two different alleles each. As the probability of an allele being absent in a random sample is $\left(1-f_{i}\right)^{\mathrm{n}}$, where $f_{i}$ is the allele frequency and $n$ the bulk size, sampling 20 individuals corresponds to $0.32 \%$ probability of not detecting an allele present at a frequency of 0.25 in the hybrid. Thus, it is highly recommended to use a representative bulk of individuals in order to ensure the sampling reliability required for DUS tests.

\section{Characterization of the SSR marker set}

Twenty microsatellites selected based on PIC values and genome distribution were converted to the UTSP system to genotype 48 commercial maize hybrids registered in Brazil. A high level of polymorphism was found for the 20 SSR loci, with an average of 9.8 alleles per locus, ranging from four alleles for phi116 and umc1862 to 19 for umc1016. The PIC value ranged from 0.59 for umc1862 to 0.97 for bnlg1006, with an average of 0.84 (Table 3), which were relatively high compared with SSR markers described for apple cultivars $(0.72$, Galli et al., 2005), rice hybrids (0.35, Hashemi et al., 2009) and commercial maize hybrids (0.51, Daniel et al., 2012). These high values compared to other studies can be partially explained by the previous selection of the SSR markers based on their PIC value, but may also reflect the large genetic variability of maize cultivars in the Brazilian market. Garcia et al. (2004) genotyped a set of 18 tropical maize inbred lines with different molecular markers, including SSRs, which presented a mean PIC value of 0.89 . Besides the selection of highly informative SSRs, the tropical maize lines evaluated in this study also present wide genetic variability.

Table 2 - Estimated size in base pairs of the amplified fragments of three hybrids and their parents for SSRs umc1016, umc1033 and umc1653 using the universal tail sequence primers (UTSP) and the conventional fluorescently labeled primers (Conv.) in capillary electrophoresis.

\begin{tabular}{|c|c|c|c|c|c|c|}
\hline \multirow{2}{*}{ Genotype } & \multicolumn{2}{|c|}{ umc1016 } & \multicolumn{2}{|c|}{ umc1033 } & \multicolumn{2}{|c|}{ umc1653 } \\
\hline & UTSP & Conv. & UTSP & Conv. & UTSP & Conv. \\
\hline Line $A$ & 114 & 97 & 149 & 132 & $122 / 126$ & $106 / 110$ \\
\hline Line B & 104 & 87 & 142 & 124 & 114 & 98 \\
\hline Line $C$ & 130 & 114 & 97 & 80 & 114 & 98 \\
\hline Line D & 114 & 97 & 142 & 124 & 126 & 110 \\
\hline Hybrid $(A \times B)$ & $104 / 114$ & $87 / 97$ & $142 / 149$ & $124 / 132$ & $114 / 122 / 126$ & $98 / 106 / 110$ \\
\hline Hybrid $(A \times C)$ & $114 / 130$ & $97 / 114$ & $97 / 149$ & $80 / 132$ & $114 / 122 / 126$ & $98 / 106 / 110$ \\
\hline Hybrid $(C \times D)$ & $104 / 114$ & $87 / 97$ & 142 & 124 & $114 / 126$ & $98 / 110$ \\
\hline
\end{tabular}


The genotyping system using 20 conventional SSR loci would require 20 fluorescently labeled primers and 20 regular primers, whereas under the proposed UTSP method, only three fluorescently labeled primers and 40 regular primers are needed. As fluorescently labeled primers are approximately 10 times more expensive than regular ones, using the proposed set of primers under the UTSP method reduces the total primer cost approximately by half. In addition, the three fluorescently labeled primers under the UTSP method can be used with different sets of primers even for unrelated species due to the non-specificity of their sequences, which reduces even more the genotyping costs.

\section{Molecular identity test}

The set of 20 SSR markers generated a unique allelic combination for each single-cross hybrid evaluated, with the probability of random identity values ranging from $10^{-14}$, for hybrids AS1555YG, AS1572YG and Formula, to $10^{-7}$ for hybrid Garra (Table 4). These values generated a minimum exclusion power of $99.99998 \%$ (Table 4). Six SSR markers generated an overall exclusion power greater than $99.99 \%$ in a group of 192 eucalyptus trees (Kirst et al., 2005), whereas 13 SSR markers conferred more than 99.9999 $\%$ of exclusion power to distinguish 32 soybean cul-

Table 3 - Chromosome position in bin, repeat sequence motif, allelic range in base pairs (bp), number of alleles and polymorphic information content (PIC) for the 20 SSR markers based on the universal tail sequence primers (UTSP) method in 48 Brazilian commercial maize hybrids.

\begin{tabular}{lccccc}
\hline SSR & Bin & Motif & $\begin{array}{c}\text { Allelic range } \\
(\mathrm{bp})^{*}\end{array}$ & $\begin{array}{c}\text { Number of } \\
\text { alleles }\end{array}$ & PIC \\
\hline bnlg1006 & 5.00 & AG & $164-291$ & 12 & 0.97 \\
bnlg125 & 2.02 & AG & $313-422$ & 7 & 0.84 \\
bnlg1338 & 2.01 & AG & $166-280$ & 8 & 0.82 \\
bnlg161 & 6.00 & AG & $118-223$ & 11 & 0.87 \\
bnlg182 & 1.03 & AG & $83-401$ & 13 & 0.87 \\
bnlg1863 & 8.03 & AG & $114-182$ & 11 & 0.87 \\
bnlg2241 & 3.06 & AG & $188-244$ & 6 & 0.77 \\
bnlg589 & 4.10 & AG & $134-212$ & 6 & 0.95 \\
dupssr14 & 8.09 & CT/CA & $65-132$ & 8 & 0.84 \\
dupssr24 & 2.08 & GA & $75-147$ & 11 & 0.90 \\
phi053 & 3.05 & ATAC & $161-192$ & 6 & 0.74 \\
phi084 & 10.04 & GAA & $88-160$ & 9 & 0.84 \\
phi116 & 7.06 & ACTG/ACG & $136-170$ & 4 & 0.74 \\
umc1008 & 4.01 & GT/GA & $152-175$ & 8 & 0.81 \\
umc1016 & 7.02 & CT & $68-233$ & 19 & 0.92 \\
umc1019 & 5.06 & CT & $53-105$ & 18 & 0.92 \\
umc1033 & 9.02 & GA & $71-189$ & 16 & 0.92 \\
umc1084 & 10.07 & CT & $69-163$ & 9 & 0.77 \\
umc1653 & 6.07 & GAAA & $87-237$ & 10 & 0.87 \\
umc1862 & 1.11 & GA & $121-150$ & 4 & 0.59 \\
\hline Mean & & & & 9.8 & 0.84 \\
\hline *The allelic range was considered withoutthe extra 17 bp tail. &
\end{tabular}

*The allelic range was considered without the extra $17 \mathrm{bp}$ tail. tivars (Oliveira et al., 2010). Thus, this set of 20 SSR markers confers a high distinctiveness capacity and could be recommended as a molecular descriptor for maize cultivar registration purposes.

\section{Genetic diversity}

A classical MDS biplot was constructed to represent the genetic relationship of the 48 maize commercial hybrids (Figure 1). Single-cross hybrids from the same company tended to group together, as expected, whereas other genotypes showed a wider dispersion, such as the hybrids from Coodetec along the first coordinate, and the hybrids from Pioneer along the second coordinate. Coordinates 1 and 2 explained $17 \%$ and 11 $\%$ of the total variance, respectively. For a better representation of the genetic relationship among hybrids, a higher number of dimensions (coordinates) would be required to increase the percentage of variance explained in the MDS analysis. Moreover, the different number of genotypes sampled for each seed company impairs a precise inference about the genetic diversity of the commercial hybrids. Despite these limitations, the current MDS analysis was able to represent the genetic diversity of the 48 Brazilian commercial maize hybrids.

\section{Conclusion}

The set of 20 SSR markers based on the UTSP method was efficient and cost-effective for molecular fingerprinting of single-cross maize hybrids. The proposed method reduces both the genotyping costs and time when compared to the conventional SSR marker, and presents a great power of distinctiveness. Thus, this genotyping system can be applied to genetic purity testing and as a molecular descriptor for maize cultivar registration.

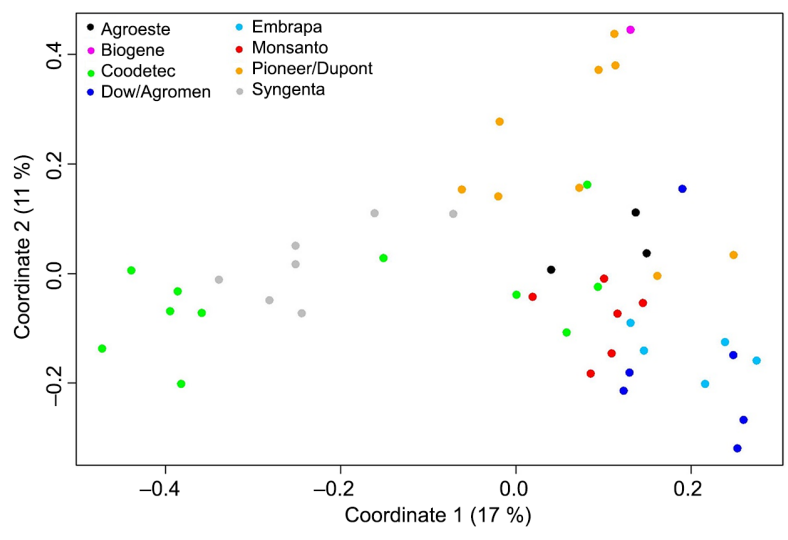

Figure 1 - Classical multidimensional scaling (MDS) biplot constructed based on 20 SSR markers obtained via the universal tail sequence primers (UTSP) method for 48 maize commercial hybrids. The percentage of variance explained by each coordinate is indicated within brackets. 
Table 4 - Probability of random identity (PRI) and the percentage of exclusion power (EP) of the 20 SSR markers for each of the 48 Brazilian commercial maize hybrids.

\begin{tabular}{|c|c|c|c|c|c|c|c|}
\hline Company & Hybrid & PRI & $\mathrm{EP}(\%)$ & Company & Hybrid & PRI & EP (\%) \\
\hline Agroeste & AS1555YG & $5.5521 \mathrm{e}-14$ & >99.9999999 & Embrapa & BRS1035 & $5.5176 \mathrm{e}-08$ & 99.99999 \\
\hline Agroeste & AS1572YG & $8.5328 \mathrm{e}-14$ & >99.9999999 & Embrapa & BRS1040 & $1.9607 \mathrm{e}-10$ & $>99.999999$ \\
\hline Agroeste & AS1590YG & $2.4200 \mathrm{e}-10$ & >99.9999999 & Monsanto & AG5055 & $2.8105 \mathrm{e}-10$ & >99.999999 \\
\hline Biogene & BG7060YG & $6.3911 \mathrm{e}-10$ & >99.9999999 & Monsanto & AG7088 & $2.9879 \mathrm{e}-11$ & >99.999999 \\
\hline Coodetec & CD316 & $4.7074 \mathrm{e}-13$ & >99.999999 & Monsanto & AG8088YG & $2.5283 e-09$ & >99.999999 \\
\hline Coodetec & CD321 & $2.7254 \mathrm{e}-10$ & >99.9999999 & Monsanto & DKB240YG & $2.1739 \mathrm{e}-11$ & >99.999999 \\
\hline Coodetec & CD327 & $6.9588 \mathrm{e}-12$ & >99.9999999 & Monsanto & DKB370 & $2.0282 \mathrm{e}-11$ & >99.999999 \\
\hline Coodetec & CD351 & $6.6698 \mathrm{e}-12$ & >99.9999999 & Monsanto & DKB390PR09 & $9.8909 \mathrm{e}-11$ & >99.999999 \\
\hline Coodetec & CD352 & $8.1453 \mathrm{e}-13$ & >99.9999999 & Pioneer/Dupont & P3646H & $1.3091 \mathrm{e}-11$ & >99.999999 \\
\hline Coodetec & CD353 & $2.0678 \mathrm{e}-10$ & >99.9999999 & Pioneer/Dupont & P3862H & $6.4119 \mathrm{e}-12$ & >99.999999 \\
\hline Coodetec & CD355 & $2.2702 \mathrm{e}-11$ & >99.9999999 & Pioneer/Dupont & P4285 & $3.7538 \mathrm{e}-11$ & >99.9999999 \\
\hline Coodetec & CD356 & $8.6811 \mathrm{e}-08$ & 99.99999 & Pioneer/Dupont & $30 F 35 \mathrm{H}$ & $6.6065 \mathrm{e}-12$ & >99.999999 \\
\hline Coodetec & CD378 & $1.5299 \mathrm{e}-12$ & >99.9999999 & Pioneer/Dupont & $30 F 53$ & $3.8697 \mathrm{e}-13$ & >99.999999 \\
\hline Coodetec & CD388 & $2.2654 \mathrm{e}-08$ & >99.9999999 & Pioneer/Dupont & $30 R 50$ & $2.6037 \mathrm{e}-12$ & >99.999999 \\
\hline Coodetec & CD397 & $1.4734 \mathrm{e}-10$ & >99.9999999 & Pioneer/Dupont & $30 S 31$ & $4.2427 \mathrm{e}-13$ & >99.999999 \\
\hline Dow & 2B587HX & $2.8918 \mathrm{e}-10$ & >99.9999999 & Pioneer/Dupont & $30 S 40$ & $3.8683 e-09$ & >99.999999 \\
\hline Dow & 2B707HX & $2.1619 \mathrm{e}-12$ & >99.9999999 & Pioneer/Dupont & $32 \mathrm{R} 48$ & $2.8428 \mathrm{e}-12$ & >99.999999 \\
\hline Dow & 2B710HX & $4.4822 \mathrm{e}-11$ & >99.9999999 & Syngenta & Formula & $1.9571 \mathrm{e}-14$ & >99.999999 \\
\hline Dow/Agromen & $20 \mathrm{~A} 55 \mathrm{HX}$ & $8.8706 \mathrm{e}-10$ & >99.999999 & Syngenta & Garra & $2.2203 e-07$ & 99.99998 \\
\hline Dow/Agromen & $20 A 68$ & $1.6273 \mathrm{e}-11$ & >99.9999999 & Syngenta & Impacto & $3.4605 \mathrm{e}-12$ & >99.999999 \\
\hline Dow/Agromen & 30A86HX & $8.0089 \mathrm{e}-12$ & >99.9999999 & Syngenta & Maximus TL & $4.2629 \mathrm{e}-11$ & >99.999999 \\
\hline Embrapa & BRS1001 & $4.5720 \mathrm{e}-11$ & >99.999999 & Syngenta & Penta TL & $1.9610 \mathrm{e}-09$ & >99.999999 \\
\hline Embrapa & BRS1010 & $1.2760 \mathrm{e}-11$ & >99.9999999 & Syngenta & Status TL & $3.8622 \mathrm{e}-11$ & >99.999999 \\
\hline Embrapa & BRS1030 & $1.1475 \mathrm{e}-09$ & >99.999999 & Syngenta & Tork & $9.4027 \mathrm{e}-13$ & >99.999999 \\
\hline
\end{tabular}

\section{Acknowledgements}

The authors are thankful to the Brazilian National Council for Scientific and Technological Development (CNPq) and the Brazilian Agency of Innovation (Finep) for their financial support, to the AgroGenética company and the Brazilian Agricultural Research Corporation (Embrapa) for their logistical support.

\section{References}

Arruda, M.P.; Goncalves, E.C.; Schneider, M.P.C.; Silva, A.L.D.; Morielle-Versute, E. 2010. An alternative genotyping method using dye-labeled universal primer to reduce unspecific amplifications. Molecular Biology Reports 37: 2031-2036.

Associação Paulista dos Produtores de Sementes e Mudas [APPS]. 2015. Statistical Survey of Maize Seed Market $=$ Levantamento Estatístico do Mercado de Sementes de Milho. Atualizado até $1^{\circ}$ Levantamento Verão 2015. Available at: apps.agropecuaria. ws/site/Relatorio/30 [Accessed Aug 20, 2015] (in Portuguese).
Cryer, N.C.; Butler, D.R.; Wilkinson, M.J. 2005. High throughput, high resolution selection of polymorphic microsatellite loci for multiplex analysis. Plant Methods 1: 3.

Daniel, I.O.; Adetumbi, J.A.; Oyelakin, O.O.; Olakojo, S.A.; Ajala, M.O.; Onagbesan, S.O. 2012. Application of SSR markers for genetic purity analysis of parental inbred lines and some commercial hybrid maize (Zea mays L.). American Journal of Experimental Agriculture 2: 597-606.

Diniz, F.M.; Iyengar, A.; Lima, P.S.D.; MacLean, N.; Bentzen, P. 2007. Application of a double-enrichment procedure for microsatellite isolation and the use of tailed primers for high throughput genotyping. Genetics and Molecular Biology 30: 380-384.

Galli, Z.; Halasz, G.; Kiss, E.; Heszky, L.; Dobranszki, J. 2005. Molecular identification of commercial apple cultivars with microsatellite markers. Hortscience 40: 1974-1977.

Garcia, A.A.F.; Benchimol, L.L.; Barbosa, A.M.M.; Geraldi, I.O.; Souza, C.L.; Souza, A.P. 2004. Comparison of RAPD, RFLP, AFLP and SSR markers for diversity studies in tropical maize inbred lines. Genetics and Molecular Biology 27: 579-588. 
Hashemi, S.H.; Mirmohammadi-Maibody, S.A.M.; Nematzadeh, G.A.; Arzani, A. 2009. Identification of rice hybrids using microsatellite and RAPD markers. African Journal of Biotechnology 8: 2094-2101.

Hayden, M.J.; Nguyen, T.M.; Waterman, A.; Chalmers, K.J. 2008. Multiplex-ready PCR: a new method for multiplexed SSR and SNP genotyping. BMC Genomics 9: 80.

Jesus, O.N.; Ferreira, C.F.; Silva, S.D.E.; Camara, T.R.; Soares, T.L.; Pestana, K.N. 2009. Characterization of recommended banana cultivars using morphological and molecular descriptors. Crop Breeding and Applied Biotechnology 9: 164-173.

Kirst, M.; Cordeiro, C.M.; Rezende, G.D.S.P.; Grattapaglia, D. 2005. Power of microsatellite markers for fingerprinting and parentage analysis in Eucalyptus grandis breeding populations. Journal of Heredity 96: 161-166.

Liu, K.; Goodman, M.; Muse, S.; Smith, J.S.; Buckler, E.; Doebley, J. 2003. Genetic structure and diversity among maize inbred lines as inferred from DNA microsatellites. Genetics 165: 21172128.

Ministério da Agricultura, Pecuária e Abastecimento [MAPA]. 2015. National Service of Cultivar Protection = Serviço Nacional de Proteção de Cultivar. Available at: http://www. agricultura.gov.br [Accessed Jan 7, 2015] (in Portuguese).

Missiaggia, A.; Grattapaglia, D. 2006. Plant microsatellite genotyping with 4-color fluorescent detection using multipletailed primers. Genetics and Molecular Research 5: 72-78.

Nei, M.; Li, W.H. 1979. Mathematical model for studying genetic variation in terms of restriction endonucleases. Proceedings of the National Academy of Sciences 76: 5269-5273.
Oetting, W.S.; Lee, H.K.; Flanders, D.J.; Wiesner, G.L.; Sellers, T.A.; King, R.A. 1995. Linkage analysis with multiplexed short tandem repeat polymorphisms using infrared fluorescence and M13 tailed primers. Genomics 30: 450-458.

Oliveira, M.B.; Vieira, E.S.N.; Schuster, I. 2010. Construction of a molecular database for soybean cultivar identification in Brazil. Genetics and Molecular Research 9: 705-720.

Priolli, R.H.G.; Mendes-Junior, C.T.; Arantes, N.E.; Contel, E.P.B. 2002. Characterization of Brazilian soybean cultivars using microsatellite markers. Genetics and Molecular Biology 25: 185-193

Ribeiro, C.A.G.; Tanure, J.P.M.; Maciel, T.E.F.; Barros, E.G. 2013. Molecular characterization of soybean cultivars by microsatellite markers with universal tail sequence. Pesquisa Agropecuária Brasileira 48: 270-279.

Rodrigues, D.H.; Neto, F.D.; Schuster, I. 2008. Identification of essentially derived soybean cultivars using microsatellite markers. Crop Breeding and Applied Biotechnology 8: 74-78.

Saghai-Maroof, M.A.; Soliman, K.M.; Jorgensen, R.A.; Allard, R.W. 1984. Ribosomal DNA spacer-length polymorphisms in barley: mendelian inheritance, chromosomal location, and population dynamics. Proceedings of the National Academy of Sciences 81: 8014-8018.

Salgado, K.C.P.C.; Vieira, M.G.G.C.; Pinho, E.V.R.V.; Guimarães, C.T.; Pinho, R.G.V.; Sousa, L.V. 2006. Genetic purity certificate in seeds of hybrid maize using molecular markers. Revista Brasileira de Sementes 28: 169-175. 